$5 \% 5$-FU and 90\% TCA ( $\mathrm{p}=0,274)$. Subjective side effect in $1 \%$ 5-FU was significantly milder than 90\% TCA $(p=0,004)$, but the significant milder objective side effect only found at week 2,6, and $7(\mathrm{p}<0,05)$. Meanwhile subjective side effect in $5 \%$ 5-FU was also significantly milder than 90\% TCA $(p=0,001)$, but the significant milder objective side effect only found at week $2(\mathrm{p}=0,000)$.

Conclusion 1\% 5-FU and 5\% 5-FU cream have no difference effectivity compared to $90 \%$ TCA. Regarding the side effect, $1 \%$ 5-FU has significantly milder than 90\% TCA. We concluded that 5-FU may become alternative topical therapy with self-application as the benefit and $1 \% 5$-FU cream is more recommended due to milder side effect.

Disclosure No significant relationships.

\section{P320 INCENTIVE TESTING AND TREATMENT FOR STBBI IN HARD TO REACH POPULATIONS IN EDMONTON, ALBERTA, CANADA}

${ }^{1}$ John Niruban, ${ }^{2}$ Garret Meyer, ${ }^{2}$ Penny Parker, ${ }^{1}$ Jennifer Gratrix, ${ }^{3}$ Petra Smyczek*. ${ }^{1}$ Alberta Health Services, Centralized STI Services, Edmonton, Canada; ${ }^{2}$ Alberta Health Services, STI Clinic, Edmonton, Canada; ${ }^{3}$ Alberta Health Services, STI Services, Edmonton, Canada

10.1136/sextrans-2019-sti.431

Background Since 2014, Edmonton, Alberta, Canada has seen an alarming rise in infectious syphilis and gonorrhea infections. Individuals from vulnerable communities with substance use, involvement with corrections, transactional sex, and inadequately housed are overrepresented among cases. The aim of this project was to increase access to sexually transmitted and blood borne infections (STBBI) testing and treatment among hard to reach populations in Edmonton.

Methods Outreach teams from the Edmonton STI clinic consisting of a registered nurse and community health representative or licensed practical nurse offered STBBI testing at subsidized housing locations, community based organizations, and through street outreach. Clients were offered testing and treatment for chlamydia (CT), gonorrhea (NG), syphilis, HIV, Hepatitis C. Clients received a $\$ 10$ gift card for testing and a $\$ 10$ gift card when returning for results and/or treatment.

Results From October 2018 to February 2019, 393 testing visits were completed among 342 individuals. Nearly two-thirds $(61 \% ; n=207)$ of individuals were men with a median age of 32 years. Women were younger with a median age of 20.4 years. Nearly $60 \% \quad(57.9 \% ; n=198)$ of individuals reported substance use with $19.0 \% \quad(n=65)$ reporting injection drug use. Six percent $(n=20)$ of individuals were involved in transactional sex. The positivity rate for CT was 9.5\% $(n=26)$ and $4.0 \%(\mathrm{n}=11)$ for NG (273 tested). The positivity rate for HCV was $5.4 \%(n=15 ; 278$ tested). The syphilis seropositivity rate was $10.8 \%(n=34 ; 315$ tested). No new HIV cases were found. Eight-percent $(n=31)$ of visits involved treatment for an ST

Conclusion Offering STBBI incentivized testing was effective in improving access to testing and treatment for hard to reach clients resulting in high positivity rates for STBBI. By offering testing and treatment to individuals linked to high transmission activities, we aim to reduce the burden of STBBI among vulnerable groups

Disclosure No significant relationships.

\section{P321 THE SEXUAL AND REPRODUCTIVE HEALTH NEEDS OF THE HARD TO REACH POPULATIONS IN UGANDA}

Samuel Mukasa*. Theta Uganda, Programs, Kampala, Uganda

\subsection{6/sextrans-2019-sti.432}

Background Despite efforts by Ministry of Health (MOH) and implementing partners (IPs) to implement programs that are complementary to static services, some communities remain hard to reach and sustain low access to existing SRH services. Majority live in places where there are inadequate health services or are hard to reach within the general public and are underserved by the existing SRH services (de paz et al, 2014). Majority of these groups were displaced from their indigenous habitats in the $20^{\text {th }}$ century but remain hard to reach due to factors like; geographical location, cultural beliefs, nomadic life style and biological factors. Majority suffer from attacks from neighbors, are isolated and continue to be underserved by existing service structures. As a result, there's been minimal change in SRH indicators over the past ten years despite growing focus by IPs.

Methods Qualitative design utilizing case study approach to qualitative inquiry

Results The SRHR needs of the hard to reach groups are similar though with varying levels of severity among the different groups but of greater impact in these marginalized communities compared to the general public. The key SRHR needs include; STIs, SGBV, family planning, Female Genital Mutilation, Health facility deliveries, low ANC attendance and the role of TBAs. The most significant barriers include; high levels of extreme poverty, poor cultural beliefs and practices, low literacy levels, alcohol abuse, language barriers, early marriages, poor health systems and distance between the clients and available health services

Conclusion Majority of the SRH needs are known in the existing literature and not unique to hard to reach groups. These needs have greater impacts among the hard to reach groups compared to the general public. The key barriers to SRHR services are; language barrier with neighbouring societies, poor cultural beliefs and practices, poverty and long distance to existing health services

Disclosure No significant relationships.

\section{P322 A BRIEF CLINIC-BASED PEER-TO-PEER EDUCATION INTERVENTION TO IMPROVE PREVENTION PRACTICES AMONG SEXUAL MINORITY MALES}

${ }^{1}$ Nathan Lachowsky*, ${ }^{1}$ Karyn Fulcher, ${ }^{1}$ Allan Lal, ${ }^{2}$ Richard Crosby. ${ }^{1}$ University of Victoria, School of Public Health and Social Policy, Victoria, Canada; ${ }^{2}$ University of Kentucky, Lexington, USA

\subsection{6/sextrans-2019-sti.433}

Background Gay, bisexual, and other men who have sex with men (GBM) are disproportionately affected by STIs and HIV. Originally efficacious with young Black GBM, Focus on the Future $(\mathrm{FoF})$ is a clinic-based, single session intervention aimed at improving prevention practices. We examined the efficacy of the program when adapted for Vancouver's ethnoracially diverse GBM communities. 